\title{
Prevalência da Lipodistrofia Associada ao HIV em Pacientes Ambulatoriais Brasileiros: Relação com Síndrome Metabólica e Fatores de Risco Cardiovascular
}

artigo original

Leandro A. DieHL

JANAÍNA R. DIAS

Aline C. S. PAES

MARIA C. THOMAZINI

LORENA R. GARCIA

EDUARDO CINAGAWA

SUSANA L. WIECHMANN

Alexandre J. F. Carrilho

Centro de Ciências do Departamento de Clínica Médica da Saúde da Universidade Estadual de Londrina (CCS/UEL), PR, Brasil.

Recebido em 10/08/2007 Aceito em 03/04/2008

\section{RESUMO}

A lipodistrofia associada ao HIV (LAHIV) acomete $40 \%$ a $50 \%$ dos pacientes infectados pelo vírus, mas sua prevalência no Brasil é desconhecida. O objetivo deste trabalho foi avaliar a prevalência de LAHIV entre adultos brasileiros infectados, bem como sua relação com fatores de risco cardiovascular e síndrome metabólica (SM). Foram avaliados 180 pacientes maiores de 18 anos, infectados por HIV, atendidos no Ambulatório de Infectologia da Universidade Estadual de Londrina. Por meio de entrevista e revisão de prontuário, foram avaliados dados antropométricos, pressão arterial, antecedentes mórbidos pessoais e familiares, duração da infecção por HIV e da aids, drogas anti-retrovirais utilizadas, CD4+, carga viral, glicemia e perfil lipídico. A LAHIV foi definida como a presença de alterações corporais percebidas pelo próprio paciente e confirmadas ao exame clínico. A SM foi diagnosticada usando os critérios do Adult Treatment Panel III (NCEP-ATPIII), revistos e atualizados pela American Heart Association (AHA/NHLBI). A prevalência observada de LAHIV foi de $55 \%$. Os pacientes com LAHIV apresentaram maior duração da infecção por HIV, da aids e do uso de anti-retrovirais. Na análise multivariada, estiveram independentemente associados ao risco de LAHIV: sexo feminino $(p=0,006)$ e duração da aids $\geq 8$ anos $(p<0,001)$. Quanto aos critérios para SM, hipertensão foi detectada em $32 \%$, baixo HDL-colesterol em $68 \%$, hipertrigliceridemia em $55 \%$, cintura aumentada em $17 \%$ e glicemia aumentada e/ou diabetes em $23 \%$ dos indivíduos. A cintura aumentada e a hipertrigliceridemia foram mais comuns em portadores de LAHIV. A SM foi identificada em $36 \%$ dos pacientes. Na análise multivariada, estiveram associados à $\mathrm{SM}$ : IMC $\geq$ $25 \mathrm{~kg} / \mathrm{m}^{2}(\mathrm{p}<0,001)$, história familiar de obesidade $(\mathrm{p}=0,01)$, uso de indina$\operatorname{vir}(p=0,001)$ e idade $\geq 40$ anos no diagnóstico do HIV $(p=0,002)$. A LAHIV apresentou tendência a ser mais comum em portadores de SM $65 \%$ versus $50 \%, p=0,051$ ). A prevalência de LAHIV que se observou neste grupo (55\%) foi similar à descrita em estudos prévios de outros países. A prevalência de SM nestes pacientes parece ser diferente da descrita em adultos brasileiros não-infectados pelo HIV. (Arq Bras Endocrinol Metab 2008; 52/4:658-667)

Descritores: Síndrome da lipodistrofia associada ao HIV; Síndrome X metabólica; Infecções por HIV; Prevalência; Brasil.

\section{ABSTRACT}

Prevalence of HIV-Associated Lipodystrophy in Brazilian Outpatients: Relation with Metabolic Syndrome and Cardiovascular Risk Factors.

Lipodystrophy in HIV-infected patients (LDHIV) affects $40-50 \%$ of HIV-infected patients, but there are no data on its prevalence in Brazil. The aim of this study was to assess the LDHIV prevalence among HIV-infected adult Brazilian individuals, as well as to evaluate LDHIV association with cardiovascular risk factors and the metabolic syndrome (MS). It was included 180 adult HIV-infected outpatients consecutively seen in the Infectology Clinic of Universidade Estadual de Londrina. Anthropometric and clinical data (blood pressure, 
family and personal comorbidities, duration of HIV infection/AIDS, antiretroviral drugs used, CD4+ cells, viral load, fasting glycemia and plasma lipids) were obtained both from a clinical interview as well as from medical charts. LDHIV was defined as the presence of body changes self-reported by the patients and confirmed by clinical exam. MS was defined using the NCEP-ATPIII criteria, reviewed and modified by AHA/NHLBI. A 55\% prevalence of LDHIV was found. Individuals with LDHIV presented a longer infected period since HIV infection, longer AIDS duration and longer use of antiretroviral drugs. In multivariate analysis, women $(p=0.006)$ and AIDS duration $\geq 8$ years $(p<0.001)$ were independently associated with LDHIV. Concerning MS diagnostic criteria, high blood pressure was found in $32 \%$, low HDL-cholesterol in $68 \%$, hypertriglyceridemia in $55 \%$, altered waist circumference in $17 \%$ and altered glycemia and/or diabetes in $23 \%$ of individuals. Abnormal waist and hypertriglyceridemia were more common in LDHIV-affected individuals. MS was diagnosed in $36 \%$. In multivariate analysis, the factors associated with MS were: $\mathrm{BMI} \geq 25 \mathrm{Kg} / \mathrm{m}^{2}(p<0.001)$, family history of obesity $(p=0.01)$, indinavir $(p=0.001)$ and age $\geq 40$ years on HIV first detection $(p=0.002)$. There was a trend to higher frequency of LDHIV among patients with MS $(65 \%$ versus $50 \%, p=0.051)$. LDHIV prevalence among our patients $(55 \%)$ was similar to previous reports from other countries. MS prevalence in these HIV-infected individuals seems to be similar to the prevalence reported on Brazilian nonHIV-infected adults. (Arq Bras Endocrinol Metab 2008; 52/4:658-667)

Keywords: HIV-associated lipodystrophy syndrome; Metabolic syndrome X; HIV infections; Prevalence; Brazil.

\section{INTRODUÇÃO}

Osing INÍCIO DO TRATAMENTO DA SÍNDROME da imunodeficiência humana (aids) com a terapia anti-retroviral altamente ativa (TARV), na década de 1990, aumentou consideravelmente a duração e a qualidade de vida dos portadores da doença. Porém, a redução da morbimortalidade por doenças infecciosas e neoplásicas oportunistas vem se acompanhando do aumento da prevalência de outras patologias, entre elas a lipodistrofia associada ao HIV (LAHIV), um quadro clínico composto por alterações endócrino-metabólicas complexas que pode estar associado a significativo aumento de risco cardio$\operatorname{vascular}(1)$.

A LAHIV parece estar associada a fatores de risco, como: idade, peso, sexo e uso de inibidores de protease (2). A prevalência de lipodistrofia, avaliada em diversos estudos, variou de $18 \%$ a $83 \%$ (3). Um estudo brasileiro relatou que a prevalência de alterações corporais compatíveis com lipodistrofia foi de $65 \% \mathrm{em}$ pacientes infectados por HIV em acompanhamento ambulatorial, e positivamente relacionada ao uso dos anti-retrovirais (4). Não há consenso sobre a forma de diagnóstico mais apropriada para a LAHIV, mas os critérios mais utilizados atualmente são essencialmente clínicos e incluem o relato do paciente de alterações na distribuição de gordura corporal (acúmulo de gordura em tronco e/ou perda de tecido adiposo em face e membros), associado à confirmação dessas alterações pela inspeção do médico (1).

A síndrome metabólica (SM), por outro lado, é um conjunto de anormalidades metabólicas relacionadas ao excesso de adiposidade visceral, cuja importância está bem consolidada na literatura e na prática médica, apesar de haver algumas divergências em relação ao diagnóstico em razão da existência de várias definições, propostas por diferentes sociedades (5). A SM e a LAHIV compartilham algumas características comuns, como distribuição anormal de gordura corpórea, resistência à insulina, anormalidades lipídicas e, possivelmente, aumento de risco cardiovascular.

A prevalência de $S M$ em pacientes infectados por HIV em uso de TARV foi estimada em $16 \%$ a $21 \%$, em estudos realizados no exterior (6-8). Entretanto, não encontramos dados sobre a prevalência de LAHIV e de SM em pacientes brasileiros infectados pelo HIV. O presente trabalho foi desenvolvido com o objetivo de determinar a prevalência de LAHIV em pacientes bra- 
sileiros infectados pelo HIV, bem como avaliar as possíveis associações entre a LAHIV, a SM e os fatores de risco cardiovascular clássicos nessa população.

\section{CASUÍSTICA E MÉTODOS}

Durante seis meses, todos os pacientes infectados por HIV, com mais de 18 anos, atendidos no Ambulatório de Infectologia/aids do Hospital de Clínicas da Universidade Estadual de Londrina (HC/UEL), foram convidados a participar do estudo. Os critérios de exclusão foram: gestação, recusa em participar do estudo, incapacidade de permanecer em posição ortostática sem ajuda e dados laboratoriais incompletos.

Todos os pacientes receberam informações a respeito do estudo e assinaram um Termo de Consentimento Livre e Esclarecido. O protocolo de pesquisa foi previamente aprovado pelo Comitê de Ética em Pesquisa da UEL (Parecer CEP/UEL n ${ }^{\circ}$ 071/2006) e foi conduzido segundo a Resolução n ${ }^{\circ}$ 196/1996 do Ministério da Saúde.

Os pacientes foram avaliados no momento anterior à consulta médica por estudantes de medicina treinados, que aplicaram entrevista padronizada e coletaram dados antropométricos. $\mathrm{Na}$ entrevista, foram obtidos: idade, sexo, história de tabagismo e antecedentes familiares de diabetes melito e hipertensão arterial. O peso corporal foi aferido em uma balança digital (Filizola ${ }^{\circledR}$ ), com o paciente descalço e usando roupas leves. A altura foi determinada em estadiômetro vertical fixo, em posição ortostática. A circunferência abdominal foi aferida na cicatriz umbilical, com fita métrica inelástica. A pressão arterial foi determinada no membro superior direito, após repouso de cinco minutos, com o paciente sentado, utilizando-se esfigmomanômetro de coluna de mercúrio.

Os exames laboratoriais (glicemia de jejum, colesterol total, triglicérides, HDL-colesterol, contagem de linfócitos T CD4+ e carga viral plasmática) foram coletados do prontuário médico, considerando-se o exame mais recente disponível no momento da avaliação. Apenas exames realizados no intervalo máximo de um ano anterior à coleta de dados foram considerados. A glicemia (método enzimático) e o perfil lipídico (colorimétrico enzimático) foram mensurados em aparelho automatizado Dimension $\mathrm{RXL}^{\odot}$ (Dade Behring). A contagem de linfócitos T CD4+ foi feita por citometria de fluxo, no sistema BD FACSCount ${ }^{\odot}$ (Becton Dickin- son). A carga viral foi quantificada por RT-PCR, usando amplicor HIV-1 Monitor ${ }^{\odot}$ (Roche).

Dados clínicos (histórico da infecção por HIV e da aids, drogas anti-retrovirais utilizadas, outros medicamentos em uso) foram coletados por meio de anotações no prontuário médico.

A LAHIV foi diagnosticada pela presença simultânea dos dois critérios seguintes: a) relato pelo próprio paciente de alterações na distribuição de gordura corporal, surgidas a partir do momento do diagnóstico da infecção por HIV; e b) detecção dessas alterações pelo médico, em exame clínico dirigido. As alterações da distribuição de gordura corporal consideradas neste estudo foram: lipohipertrofia central (acúmulo de gordura em tronco e/ou abdome, mamas, ou região cervical posterior - "giba de búfalo"), lipoatrofia periférica (rarefação do tecido adiposo em face, região cervical anterior e lateral, membros inferiores e/ou superiores ou nádegas) ou lipodistrofia mista (ambas as alterações, em graus variados).

A SM foi diagnosticada pelos critérios propostos pelo Adult Treatment Panel III (NCEP-ATPIII) (9), revistos e atualizados em 2005 pelo consenso da American Heart Association (AHA/NHLBI) (5), que exigem a presença de três ou mais das seguintes alterações: circunferência abdominal $\geq 88 \mathrm{~cm}$ (para mulheres) ou $\geq 102 \mathrm{~cm}$ (para homens); glicemia de jejum $\geq 100 \mathrm{mg} / \mathrm{dL}$ ou diagnóstico prévio de diabetes melito; pressão arterial $\geq 130 \times 85 \mathrm{mmHg}$ ou uso de terapia para hipertensão arterial; triglicérides $\geq 150 \mathrm{mg} / \mathrm{dL}$ ou uso de terapia para hipertrigliceridemia; e HDL-colesterol $<50 \mathrm{mg} /$ dL para mulheres ou $<40 \mathrm{mg} / \mathrm{dL}$ para homens ou uso de terapia para HDL baixo (5). Para fins de comparação, também foi avaliada a freqüência de cintura aumentada de acordo com os critérios da International Diabetes Federation (IDF) para populações sul-americanas $(\geq 80 \mathrm{~cm}$ para mulheres e $\geq 90 \mathrm{~cm}$ para homens) (10).

\section{Análise estatística}

Com base na prevalência de infecção pelo HIV de $0,61 \%$ entre adultos brasileiros (11), e em relatos da literatura que avaliam em cerca de $45 \%$ a freqüência de lipodistrofia em indivíduos infectados pelo $\operatorname{HIV}(2,12)$, o tamanho da amostra para determinar essa prevalência neste estudo foi estimado em 157 indivíduos, com nível de confiança de $80 \%$ e erro máximo aceitável de $5 \%$, levando-se em conta que a população da região metro- 
politana de Londrina (área de abrangência do HC/ UEL) era de 728.120 habitantes, em 2004 (13).

As variáveis contínuas são apresentadas na forma de médias \pm desvio-padrão (ou medianas e faixa de valores, para variáveis com distribuição não-paramétrica). As variáveis discretas (inclusive a freqüência de LAHIV e SM) são descritas na forma de porcentagem. Diferenças entre os subgrupos com e sem LAHIV foram testadas com o qui-quadrado (ou teste exato de Fisher, quando apropriado) para variáveis dicotômicas ou com o teste $t$ de Student (ou o teste de Mann-Whitney) para variáveis contínuas. As variáveis potencialmente associadas ao risco de LAHIV e SM foram dicotomizadas e avaliadas pelo teste qui-quadrado (ou teste exato de Fisher). As variáveis associadas à LAHIV e à SM com $\mathrm{p}<0,10$ foram submetidas à análise de regressão logística multivariada (step forward por razão de verossimilhança). Para as demais análises, foi adotado o nível de significância de $5 \%$. As análises foram realizadas no software SPSS 15.0 (SPSS Inc., Chicago, USA).

\section{RESULTADOS}

No período de 22 de junho a 23 de novembro de 2006 , 228 pacientes tiveram consultas agendadas no ambulatório de aids do HC/UEL. Destes, 30 não compareceram, quatro foram excluídos por não conseguirem ficar em pé, uma por gestação e quatro recusaram-se a participar. Portanto, foram coletados dados de 189 pacientes, dos quais nove foram excluídos em virtude da ausência de dados laboratoriais (glicemia).

Dos 180 pacientes considerados na análise, 59\% eram do sexo masculino. A média de idade foi de 42,9 $\pm 10,5$ anos; $61 \%$ apresentaram índice de massa corporal (IMC) normal. Os homens tiveram altura e peso maiores que as mulheres, mas não houve diferença em relação ao IMC. A maioria (96\%) dos pacientes já possuía o diagnóstico de aids, porquanto $87 \%$ estavam em uso atual de TARV e 70\% já haviam sido expostos a algum inibidor de protease. Os esquemas de TARV mais utilizados foram: AZT, 3TC e efavirenz e AZT (ou $\mathrm{D} 4 \mathrm{~T}$ ), 3TC e lopinavir/ritonavir (em combinação fixa). A carga viral era indetectável $(<400$ cópias $\mathrm{RNA} / \mathrm{mL})$ em $59 \%$, e detectável (mas $<10.000$ cópias $\mathrm{RNA} / \mathrm{mL}$ ) em $20 \%$. A contagem de linfócitos T CD4+ era maior que 350 células $/ \mathrm{mm}^{3}$ na maioria (52\%) dos pacientes, e $<200$ células $/ \mathrm{mm}^{3}$ em $22 \%$. As características dos pacientes são descritas na Tabela 1 .
Tabela 1. Dados antropométricos e clínicos dos pacientes estudados.

\begin{tabular}{|c|c|}
\hline $\mathrm{n}$ & 180 \\
\hline Sexo masculino (\%) & $106(58,9 \%)$ \\
\hline Altura média (m) & $1,64 \pm 0,09$ \\
\hline Peso médio (kg) & $65,44 \pm 13,97$ \\
\hline IMC médio $\left(\mathrm{kg} / \mathrm{m}^{2}\right)$ & $24,35 \pm 4,39$ \\
\hline \multicolumn{2}{|l|}{ Classificação do IMC (\%) } \\
\hline$>18,5$ (baixo peso) & 5,0 \\
\hline 18,5-24,9 (peso normal) & 61,1 \\
\hline 25-29,9 (sobrepeso) & 25,0 \\
\hline 30 ou mais (obesidade) & 8,9 \\
\hline $\begin{array}{l}\text { Tempo médio de infecção } \\
\text { pelo HIV (meses) }\end{array}$ & $102,9 \pm 49,9$ \\
\hline $\begin{array}{l}\text { Pacientes com diagnóstico } \\
\text { de aids (\%) }\end{array}$ & $173(96,1 \%)$ \\
\hline $\begin{array}{l}\text { Duração da aids (meses) } \\
(n=173)\end{array}$ & $85,2 \pm 40,5$ \\
\hline \multicolumn{2}{|l|}{ Uso de TARV } \\
\hline Nunca usaram & $11(6,1 \%)$ \\
\hline Uso prévio & $12(6,7 \%)$ \\
\hline Uso atual & $157(87,2 \%)$ \\
\hline $\begin{array}{l}\text { Tempo médio de uso da } \\
\text { TARV (meses) }(n=169)\end{array}$ & $77,3 \pm 37,8$ \\
\hline \multicolumn{2}{|l|}{$\begin{array}{l}\text { Esquemas de TARV mais usados } \\
\text { (uso atual; } n=157 \text { ) (\%) }\end{array}$} \\
\hline 2 ITRN + $1 \mathrm{IP}^{*}$ & $65(41,4 \%)$ \\
\hline 2 ITRN + 1 ITRNN & $52(33,1 \%)$ \\
\hline 2 ITRN + 2 IP & $20(12,7 \%)$ \\
\hline 2 ITRN + 1 ITRNN + 1 ou 2 IP & $17(10,8 \%)$ \\
\hline 2 ITRN & $2(1,3 \%)$ \\
\hline 2 ITRN + 2 IP + $1 \mathrm{IF}$ & $1(0,6 \%)$ \\
\hline $\begin{array}{l}\text { Carga viral (log cópias de } \\
\text { RNA/mL) }(n=180)\end{array}$ & $4,09 \pm 0,84$ \\
\hline CD4+ (células $/ \mathrm{mL})(\mathrm{n}=180)$ & $407,4 \pm 252,5$ \\
\hline
\end{tabular}

TARV = terapia anti-retroviral; ITRN = inibidor da transcriptase reversa análogo de nucleosídeo/nucleotídeo; ITRNN = inibidor da transcriptase reversa não-análogo de nucleosídeo; IP = inibidor de protease; IF = inibidor de fusão. *Incluídos nesta categoria os esquemas com 2 ITRN mais a combinação fixa lopinavir/ritonavir (Kaletra ${ }^{\circledR}$ ).

\section{Alterações da distribuição de gordura corporal e LAHIV}

$\mathrm{Na}$ entrevista, $\mathbf{5 7 \%}$ dos pacientes relataram alguma alteração do formato corporal surgida após o diagnóstico da infecção por HIV. As mulheres notaram principal- 
mente lipohipertrofia central e lipodistrofia mista (43\% e $40 \%$ dos relatos, respectivamente), enquanto os homens notaram lipoatrofia periférica, lipodistrofia mista e lipohipertrofia central com a mesma freqüência $(34 \%$, $34 \%$ e $32 \%$, respectivamente).

Ao exame clínico dirigido, $73 \%$ dos pacientes apresentavam algum tipo de redistribuição de tecido adiposo. Em $96 \%$ dos indivíduos que relataram alterações corporais, estas foram confirmadas ao exame clínico. Entretanto, apenas 76\% dos indivíduos com alterações visíveis ao exame relataram a presença dessas alterações. A falta de percepção das alterações corporais foi mais comum no sexo masculino: $32 \%$ dos homens com alterações visíveis não as relataram na entrevista, o que ocorreu com apenas $15 \%$ das mulheres com redistribuição de gordura visível $(\mathrm{p}=0,02)$. As alterações mais comuns ao exame clínico foram: lipodistrofia mista e lipohipertrofia central no sexo feminino ( $43 \%$ de cada), e lipodistrofia mista e lipoatrofia periférica no sexo masculino ( $42 \%$ e $35 \%$, respectivamente) $(\mathrm{p}=0,01)$.

A prevalência de LAHIV, definida pela redistribuição de gordura corporal presente no relato do próprio paciente e confirmada ao exame clínico, foi de $55 \%$. A LAHIV foi detectada em $68 \%$ dos indivíduos do sexo feminino e $46 \%$ dos do sexo masculino ( $\mathrm{p}=0,004$ ).

Os portadores de LAHIV apresentaram maior duração da infecção por HIV, maior duração da aids e maior tempo de uso de TARV em comparação com os pacientes sem diagnóstico de LAHIV (Tabela 2). A LAHIV foi detectada em $61 \%$ dos pacientes com $\geq 6$ anos de duração da infecção por HIV, e em 33\% dos com $<6$ anos (p $=0,002)$. A LAHIV também foi mais comum entre pacientes com oito anos ou mais de duração da aids (74\%) do que entre pacientes com $<8$ anos $(39 \%, \mathrm{p}<0,001)$. Não houve diferença na contagem de células CD4+ nem na carga viral entre pacientes com e sem LAHIV. Carga viral indetectável não se associou à LAHIV.

A LAHIV foi observada em $27 \%$ dos pacientes que nunca usaram TARV, 67\% dos usuários prévios e 56\% dos usuários atuais $(\mathrm{p}=0,10)$. Comparando-se os expostos à TARV (uso atual ou prévio) com os que nunca usaram TARV, houve tendência a maior freqüência de LAHIV nos primeiros ( $57 \%$ versus $27 \%, \mathrm{p}=0,055$ ). Dos portadores de LAHIV, 11\% não estavam em uso de TARV ( $8 \%$ usuários prévios e $3 \%$ nunca usaram). A LAHIV foi observada em $65 \%$ dos pacientes com uso de TARV por $\geq 6$ anos e em $41 \%$ dos usuários de TARV por $<6$ anos $(\mathrm{p}=0,001)$.
Tabela 2. Comparação entre os indivíduos sem LAHIV e com LAHIV.

\begin{tabular}{|c|c|c|}
\hline & $\begin{array}{l}\text { Sem LAHIV } \\
\quad(n=99)\end{array}$ & $\begin{array}{l}\text { Com LAHIV } \\
(n=81)\end{array}$ \\
\hline Sexo masculino (\%) & 70,4 & $49,5 \#$ \\
\hline Altura (m) & $1,66 \pm 0,09$ & $1,62 \pm 0,92$ * \\
\hline Peso (kg) & $67,03 \pm 15,1$ & $64,13 \pm 12,9$ \\
\hline $\mathrm{IMC}\left(\mathrm{kg} / \mathrm{m}^{2}\right)$ & $24,20 \pm 4,44$ & $24,47 \pm 4,37$ \\
\hline Idade (anos) & $42,43 \pm 9,76$ & $44,19 \pm 9,56$ \\
\hline $\begin{array}{l}\text { Duração da infecção } \\
\text { por HIV (meses) }\end{array}$ & $88,8 \pm 49,6$ & $114,6 \pm 47,3$ * \\
\hline Duração da aids (meses) & $71,8 \pm 38,9$ & $96,1 \pm 38,7^{*}$ \\
\hline Nunca usaram TARV (\%) & 9,9 & 3,0 \\
\hline Duração da TARV (meses) & $66,7 \pm 35,0$ & $85,4 \pm 38,0$ * \\
\hline $\begin{array}{l}\text { Contagem CD4+ } \\
\text { (células } / \mathrm{mL} \text { ) }\end{array}$ & $377,9 \pm 248,4$ & $431,4 \pm 254,5$ \\
\hline $\begin{array}{l}\text { Carga viral (log cópias } \\
\text { RNA/mL) }\end{array}$ & $4,10 \pm 0,76$ & $4,07 \pm 0,92$ \\
\hline $\begin{array}{l}\text { Carga viral indetectável } \\
\text { (< } 400 \text { cópias RNA/mL) (\%) }\end{array}$ & 57,5 & 59,6 \\
\hline Hipertensão arterial (\%) & 32,1 & 31,3 \\
\hline Diabetes melito (\%) & 9,8 & $6,0 \S$ \\
\hline Glicemia $\geq 100$ mg/dL (\%) & 23,8 & 23,2 \\
\hline $\begin{array}{l}\text { Cintura aumentada - } \\
\text { NCEP-ATPIII (\%) }\end{array}$ & 7,4 & $24,2 \#$ \\
\hline $\begin{array}{l}\text { Cintura aumentada - } \\
\text { IDF (\%) }\end{array}$ & 48,5 & $33,3 \#$ \\
\hline Triglicérides elevados (\%) & 46,9 & $61,6 \S$ \\
\hline Colesterol total alto (\%) & 29,6 & 35,4 \\
\hline HDL-colesterol baixo (\%) & 72,8 & 75,8 \\
\hline SM (\%) & 28,4 & 42,4 \\
\hline
\end{tabular}

Cintura aumentada - NCEP-ATPIII: $>88 \mathrm{~cm}$ para mulheres $\mathrm{e}>102 \mathrm{~cm}$ para homens. Cintura aumentada - IDF: $>80 \mathrm{~cm}$ para mulheres $e>90 \mathrm{~cm}$ para homens. ${ }^{*} \mathrm{p}<0,01$ ( $t$ de Student); \#p $<0,01$ (qui-quadrado); $\S p<0,05$ (qui-quadrado) na comparação com indivíduos sem LAHIV 
A LAHIV foi mais comum em pacientes expostos (uso atual ou prévio) a algum dos inibidores de protease $(61 \%)$ do que nos não-expostos $(41 \%, \mathrm{p}=0,01)$. Dos portadores de LAHIV, $22 \%$ jamais utilizaram inibidores de protease. Não houve diferença na freqüência de LAHIV em relação ao uso de inibidores da transcriptase reversa análogos ou não-análogos de nucleosídeo. Observou-se associação da LAHIV com o uso prévio de zalcitabina (77\% de LAHIV nos expostos e $51 \%$ nos não-expostos, $\mathrm{p}=0,01$ ) e com o uso (atual ou prévio) de indinavir: $69 \%$, comparado a $49 \%$ nos não-expostos ( $\mathrm{p}=0,01)$, além da tendência a maior freqüência de LAHIV entre os expostos ao tenofovir $(p=0,09)$. Não foi observada diferença em relação a nenhum outro anti-retroviral.

Houve tendência para maior freqüência de LAHIV entre os indivíduos diagnosticados com SM (65\%), comparado a $50 \%$ nos sem SM ( $\mathrm{p}=0,051)$.

Os fatores que se associaram à LAHIV na análise univariada (sexo, tempo de infecção pelo HIV, duração da aids, uso de TARV, duração da TARV, uso de inibidores de protease, exposição a zalcitabina, exposição a indinavir, exposição a tenofovir e SM) foram avaliados por análise multivariada, na qual permaneceram associados de maneira independente à LAHIV apenas o sexo feminino (beta 2,51, IC 95\% 1,3-4,8, p = 0,006) e a duração da aids $\geq 8$ anos (beta 3,53, IC 95\% 2,2-8,3, p < 0,001).

\section{Fatores de risco cardiovascular e síndrome metabólica}

Observou-se que $32 \%$ dos pacientes eram hipertensos, $14(8 \%)$ apresentavam diabetes melito tipo 2 (oito em dietoterapia, três em uso de hipoglicemiantes orais e três em insulinoterapia). Outros 28 pacientes (15\%) apresentavam glicemia de jejum $\geq 100 \mathrm{mg} / \mathrm{dL}$, totalizando 42 pacientes (23\%) com glicemia alterada.

Observou-se a seguinte freqüência de alterações lipídicas: HDL-colesterol baixo em 68\%; hipertrigliceridemia, em 55\%, e colesterol total > $200 \mathrm{mg} / \mathrm{dL}$, em $33 \%$. A média de HDL-colesterol foi de $38 \pm 14 \mathrm{mg} / \mathrm{dL}$. Estavam fazendo uso de hipolipemiantes 23 pacientes (13\%): 21 com fibrato (bezafibrato ou genfibrozila), um com estatina (pravastatina) e um com estatina + fibrato. Dos usuários de hipolipemiantes, 70\% apresentavam LAHIV.

A circunferência abdominal aumentada foi detectada em $17 \%$ dos pacientes, e foi mais comum no sexo feminino: $32 \%$, comparado a $6 \%$ no masculino $(\mathrm{p}<0,001)$. Se fosse adotada a definição da IDF (10), entretanto, cintura aumentada seria observada em $42 \%$ dos indivíduos, permanecendo mais comum no sexo feminino $(55 \%$ das mulheres e $32 \%$ dos homens, $\mathrm{p}=0,001$ ).

Não houve diferença na freqüência de alterações lipídicas (hipercolesterolemia, hipertrigliceridemia, HDL baixo), disglicemia ou hipertensão entre pacientes expostos ou não à TARV. Comparando portadores e nãoportadores de LAHIV, observou-se maior freqüência de cintura aumentada $(24 \%$ versus $7 \%, \mathrm{p}=0,002)$ e hipertrigliceridemia $(62 \%$ versus $47 \%, \mathrm{p}=0,04)$ nos lipodistróficos (Tabela 2).

A SM foi identificada em 65 pacientes $(36 \%)$ e as alterações metabólicas mais comuns foram hipertrigliceridemia (> 90\%), HDL baixo (> 90\%) e hipertensão arterial $(>80 \%)$. A freqüência de SM foi igual em ambos os sexos, tendo sido maior entre os indivíduos com idade $\geq 40$ anos no momento do estudo ( $43 \%$ versus $26 \%, \mathrm{p}=0,02)$. Além disso, a SM também foi mais comum entre os indivíduos que apresentavam idade $\geq 40$ anos no momento do diagnóstico da infecção por HIV (51\%) do que nos $<40$ anos na ocasião $(30 \%, \mathrm{p}=$ $0,009)$, sem diferença quanto à duração da infecção por HIV, duração da aids ou uso de TARV. Pacientes com seis anos ou mais de uso de TARV tiveram tendência a maior freqüência de SM $(p=0,09)$. A SM foi mais comum em pacientes expostos a inibidores de protease $(41 \%$, versus $24 \%$ nos não-expostos, $\mathrm{p}=0,02)$, apresentando associação com o uso prévio ou atual de indinavir ( $52 \%$, comparado a $30 \%$ nos não-expostos, $\mathrm{p}=0,004)$, além da tendência a maior ocorrência entre os expostos ao atazanavir $(\mathrm{p}=0,08)$. Não houve diferença no risco de SM em relação a outros anti-retrovirais. A freqüência de LAHIV não foi diferente quando os indivíduos foram classificados pelo número de critérios diagnósticos positivos para SM (Figura 1).

A SM foi mais comum nos indivíduos com excesso de peso (IMC $\geq 25 \mathrm{~kg} / \mathrm{m}^{2}$ ), $61 \%$, que naqueles com IMC normal $(23 \%, \mathrm{p}<0,001)$. Os pacientes com antecedentes de hipertensão arterial $(\mathrm{p}=0,04)$ e obesidade $(\mathrm{p}=0,007)$ entre familiares de primeiro grau também apresentaram SM mais freqüentemente.

Os fatores que se associaram à SM na análise univariada (idade atual, idade no diagnóstico da infecção por HIV, IMC, duração da TARV, uso de inibidores de protease, exposição a indinavir, exposição a atazanavir, história familiar de hipertensão, história familiar de obesidade LAHIV) foram submetidos à análise multivariada, na qual restaram associados de maneira independente à $\mathrm{SM}: \mathrm{IMC} \geq 25 \mathrm{~kg} / \mathrm{m}^{2}$ (beta 4,3 , IC $95 \%$ 


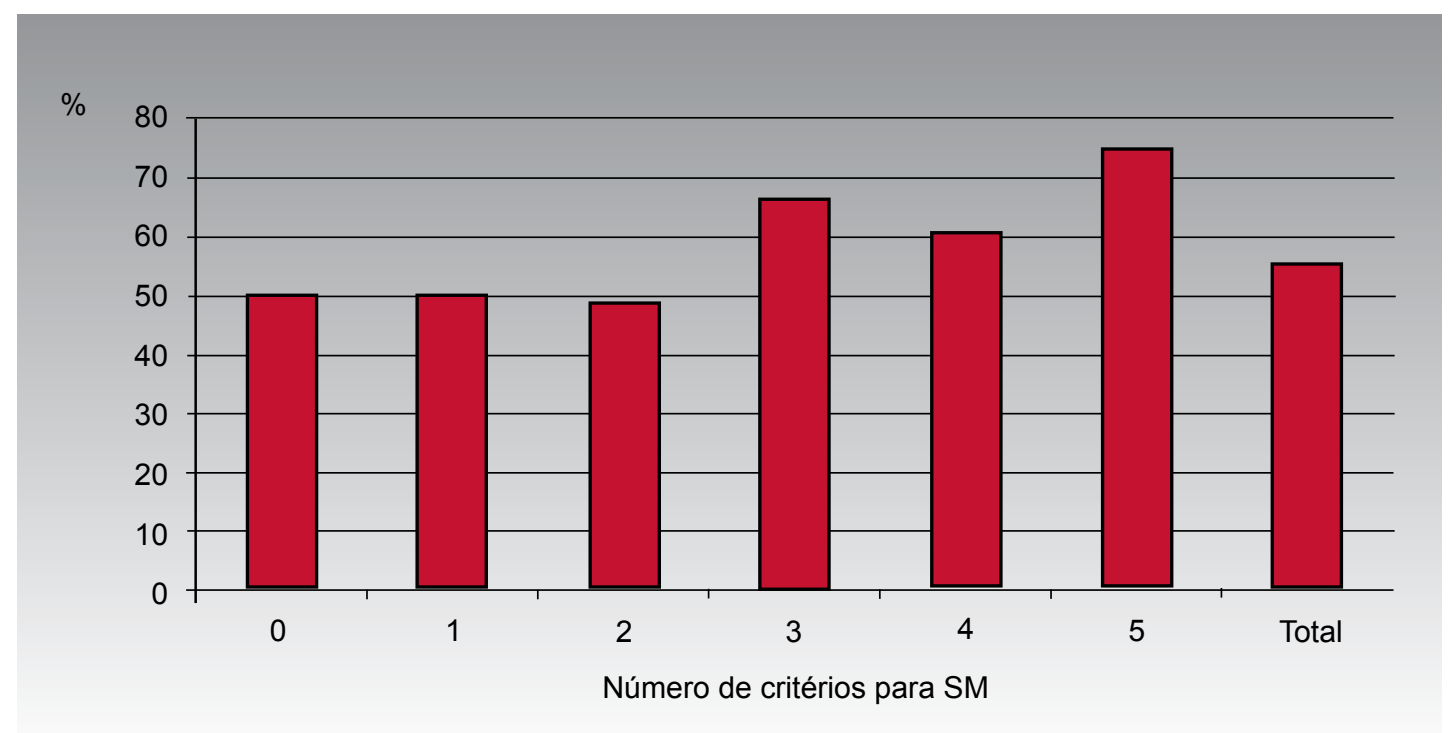

Figura 1. Freqüência (\%) de LAHIV entre os pacientes, classificados de acordo com o número de critérios diagnósticos apresentados para SM.

2,1-9,0, p $<0,001$ ), uso de indinavir (beta 3,59, IC $95 \% 1,6-7,8, \mathrm{p}=0,001)$, idade $\geq 40$ anos ao diagnóstico do HIV (beta 3,51 , IC 95\% 1,5-7,8, p = 0,002) e história familiar de obesidade (beta 2,51, IC 95\% $1,1-5,4, \mathrm{p}=0,01)$.

Os portadores de SM apresentaram risco maior de diabetes melito (OR 12,8; IC 95\% 2,6-86; $\mathrm{p}<0,001$ ) e de hipertensão arterial (OR 13,7; IC 95\% 6,4-29,3; $\mathrm{p}<0,001)$.

\section{DISCUSSÃo}

O presente estudo, até em que se tem conhecimento, é o primeiro a avaliar a prevalência de LAHIV em pacientes ambulatoriais brasileiros infectados pelo HIV. A identificação da lipodistrofia associada ao HIV é fundamental no acompanhamento de pacientes em TARV, visto que sua presença aumenta o risco de diabetes e, possivelmente, de eventos cardiovasculares $(2,14,15)$. A LAHIV parece ser mediada, mesmo antes do início da TARV, pelo aumento de citocinas inflamatórias decorrente da própria infecção pelo HIV e, mais tardiamente, também por efeitos dos anti-retrovirais (2). Os inibidores de protease inibem a proliferação/diferenciação de adipócitos e aumentam a lipólise, pela inibição do SREBP-1, bloqueando a ativação de fatores de transcrição ligados ao PPAR-gama. Já os análogos de nucleosídeos (especial- mente a estavudina) induzem disfunção mitocondrial, levando à lipoatrofia. Também são descritos distúrbios do transporte de ácidos graxos, predispondo ao acúmulo centrípeto de gordura $(2,16)$.

A prevalência de $55 \%$, que encontramos, é similar aos resultados de estudos prévios. A prevalência média dos estudos já publicados é de $42 \%(2,12,17,18)$. A grande variabilidade entre as prevalências relatadas na literatura (18\% a $83 \%)$ pode ser explicada por diferenças na definição de lipodistrofia e nas características da população estudada $(2,12)$.

O método essencialmente clínico que se adotou neste estudo para diagnóstico de lipodistrofia, apesar de mais prático e menos custoso, apresenta algumas limitações, relacionadas em maior parte à subjetividade da percepção da imagem corporal pelo paciente (2). Santos e cols., em um interessante estudo realizado em São Paulo, observaram que $65 \%$ dos pacientes infectados por HIV relatavam alterações na distribuição de gordura corporal, sendo essa freqüência maior no sexo feminino, e associada não só às características da doença (duração da infecção pelo HIV e tempo de uso de inibidores de protease), mas também a fatores psicológicos e culturais, como a qualidade do relacionamento com cônjuge/familiares e o nível de escolaridade (4). Apesar de não se ter estudado especificamente tais fatores, também observou que as mulheres relataram mais freqüentemente $o$ 
acúmulo central de gordura, enquanto os homens relataram lipoatrofia periférica e lipohipertrofia central com a mesma freqüência. Além disso, nos pacientes com redistribuição de gordura corporal evidente ao exame clínico, maior proporção de homens $(32 \%)$ que de mulheres (15\%) deixou de relatar tais alterações ao entrevistador, sugerindo que indivíduos do sexo feminino estão mais atentos a possíveis mudanças corporais, fenômeno possivelmente relacionado à maior cobrança por perfeição física imposta pela sociedade às mulheres.

Outros métodos mais objetivos para diagnóstico da lipodistrofia, como a tomografia computadorizada e a densitometria (DEXA), não são recomendados na prática clínica em virtude do custo e da exposição à radiação (2). Recentemente, um escore clínico, incorporando dados, como: sexo, idade, duração da infecção por HIV, relação cintura-quadril, HDL-colesterol, anion gap e área de tecido adiposo à tomografia, demonstrou boa acurácia para diagnóstico e mensuração da LAHIV, mas os próprios autores sugerem que sua aplicação seja mais adequada a ambientes de pesquisa que ao atendimento clínico rotineiro (19). O fato é que ainda não há consenso sobre qual o melhor método para avaliação da lipodistrofia na prática clínica (20). Acredita-se que o uso de critérios puramente clínicos, como os adotados neste estudo, tenha as vantagens de melhor aplicabilidade e baixo custo.

Os fatores associados a maior risco de lipodistrofia, descritos na literatura, são: uso de TARV, duração da TARV, uso de inibidores de protease, sexo feminino e carga viral indetectável (hipertrofia central), bem como os fatores associados especificamente com lipoatrofia: idade, baixo peso corporal pré-TARV, diagnóstico prévio de aids, menor nadir de CD4+ e uso de estavudina (2). Neste estudo, permaneceram independentemente associados à LAHIV, na análise multivariada, apenas o sexo feminino (risco 2,5 vezes maior) e a duração da aids $\geq 8$ anos (risco 4,3 vezes maior). Possivelmente, a avaliação do risco de LAHIV associada ao uso de TARV no nosso estudo tenha sido prejudicada pela pequena proporção de indivíduos sem TARV na nossa casuística (6\%). Embora a LAHIV pareça mais comum nos expostos à TARV (principalmente aos inibidores de protease), neste estudo também foram acometidos pacientes infectados por HIV não expostos a anti-retrovirais, fato já descrito por outros autores (21).

Alguns fatores de risco cardiovascular foram muito comuns na população estudada, principalmente os distúrbios lipídicos. Um grande estudo transversal relatou prevalências maiores de alterações lipídicas em indiví- duos infectados pelo HIV usando TARV (principalmente inibidores de protease) que na população geral (14). Estudos longitudinais sugerem que a dislipidemia inicia-se ainda antes da introdução da TARV (principalmente o HDL baixo), e é potencializada pelos anti-retrovirais $(22,23)$.

Em relação às alterações do metabolismo glicídico, que também foram comuns neste estudo, há evidências de que os anti-retrovirais e a redistribuição de gordura corporal aumentam o risco de resistência à insulina $\mathrm{e}$ diabetes $(1,24)$, mas, diferente da dislipidemia, não há aumento desse risco nestes pacientes antes do início da TARV (25). A freqüência de diabetes melito que observamos aproxima-se da prevalência estimada de diabetes em adultos brasileiros, 7,6\% (26). Guimarães e cols., ao estudar a sensibilidade à insulina, o perfil lipídico e a distribuição de gordura corporal (por ultrassonografia e plicometria), em pacientes de um hospital-escola de Belo Horizonte, MG (semelhante ao nosso serviço), observaram que esses parâmetros estavam alterados nos usuários de TARV, comparados aos infectados por HIV sem TARV (27). Não se pode fazer essa comparação em razão da baixa proporção de indivíduos não-expostos aos anti-retrovirais (6\%, comparados a $24 \%$ no trabalho de Guimarães e cols.) (27).

A cintura aumentada esteve presente em 17\% pelos critérios clássicos do NCEP-ATPIII $(5,9)$. Entretanto, a adoção de valores de corte mais baixos, como os propostos pela IDF, elevou essa proporção para $42 \%$ (10). Cabe ressaltar que a IDF recomenda, para a América do Sul, a adoção dos mesmos valores de corte para cintura estimados para populações sul-asiáticas, por causa da falta de dados específicos para populações sul-americanas (10). Obviamente, maiores estudos são necessários para o cálculo de valores de corte específicos para as populações latino-americanas, embora o impacto clínico do uso desses novos limiares ainda não seja claro.

A cintura aumentada e a hipertrigliceridemia foram mais comuns entre pacientes com LAHIV, corroborando o efeito da lipodistrofia na distribuição anormal de gordura corporal e no perfil lipídico $(2,3,14)$.

A freqüência de síndrome metabólica no presente estudo foi de $36 \%$. No Brasil, há muito poucos dados sobre a prevalência de SM. Oliveira e cols. estimaram que 30\% dos adultos em uma área rural do Nordeste brasileiro (não infectados por HIV, idade média 49 anos) fossem portadores de SM, pelos critérios do NCEP-ATPIII (28). Um dos primeiros estudos a avaliar SM em indivíduos com HIV foi o de Bruno e cols., que encontraram 40\% de SM 
entre 210 pacientes italianos infectados e apenas $6 \%$ nos controles não-infectados; entretanto, esses autores utilizaram a definição de SM do European Group for the Study of Insulin Resistance (EGIR), dificultando a comparação desses resultados (29). Outros estudos posteriores encontraram prevalências menores de SM, como os de Samaras e cols. na Austrália (18\% de SM pelo NCEP-ATPIII e 14\% pela IDF, entre 788 pacientes) (30; Jericó e cols. na Espanha (17\% de SM pelo NCEP-ATPIII, em 710 pacientes) (7); Estrada e cols., também na Espanha (16\% entre 146 pacientes infectados em uso de TARV, e apenas 3\% em controles não-infectados) (6); e, mais recentemente, Mondy e cols. nos Estados Unidos (26\% em infectados e controles, usando dados do NHANES) (31). O maior estudo realizado até o momento foi o de Bonfanti e cols., na Itália, que analisaram 1.263 pacientes infectados $\mathrm{e}$ 2.051 controles, e detectaram SM (pelo NCEP-ATPIII) em $21 \%$ dos pacientes com HIV e 16\% dos controles, com risco ajustado duas vezes maior de SM entre os infectados (8). A nosso ver, as discrepâncias entre os resultados dos diversos estudos não permitem, ainda, concluir se há aumento do risco de SM entre indivíduos com HIV, mas esse risco não parece estar associado à TARV ou à duração da infecção $(8,31)$.

Assim como em estudos prévios que demonstraram que o diabetes melito é cinco a nove vezes mais comum em pacientes infectados pelo HIV portadores de SM (30), em nossa casuística a SM conferiu um risco 13 vezes maior de diabetes e 14 vezes maior de hipertensão.

A associação da SM com o aumento do risco cardiovascular na população geral já foi exaustivamente demonstrada por estudos prospectivos (32). Embora haja evidências de que os pacientes com HIV apresentem maior risco de infarto do miocárdio do que a população geral (15), não há dados sobre a associação de SM com o risco cardiovascular nesses pacientes. Um estudo mostrou que a espessura íntima-média da carótida, um marcador intermediário de risco cardiovascular, estava aumentada em pacientes infectados por HIV com SM, de maneira similar à observada em diabéticos não-infectados (33). Entretanto, estudos prospectivos ainda são necessários para esclarecer o impacto da SM sobre os desfechos clínicos (eventos cardiovasculares e morte) nessa população.

Apesar de a LAHIV e a SM compartilharem várias características (distribuição anormal de gordura, resistência à insulina, dislipidemia e potencial aumento de risco cardiovascular) $(2,5)$, neste estudo, apesar de a tendência à associação das duas síndromes, esse dado não atingiu significância estatística $(\mathrm{p}=0,05 \mathrm{l})$. Nos es- tudos de Estrada e Samaras, a lipodistrofia foi mais freqüente em pacientes infectados pelo HIV com SM que entre os sem SM $(6,30)$. Bonfanti e cols. também observaram que a lipodistrofia foi mais comum entre pacientes com SM ( $54 \%$ versus $37 \%, \mathrm{p}<0,0001)$ (34). Possíveis explicações para essa diferença podem ser o menor tamanho desta amostra e a heterogeneidade destas populações. Estrada e cols., por exemplo, compararam indivíduos infectados com HIV em uso de TARV com controles saudáveis sem HIV (6).

Outros achados deste estudo merecem atenção, como a associação da SM com a idade ao diagnóstico da infecção pelo HIV (dado que não havia sido descrito previamente) e com o uso de indinavir, um inibidor de protease que já demonstrou reduzir a sensibilidade à insulina (35) e a expressão de GLUT-4 in vitro (36). A SM também se associou ao sobrepeso (risco 4 vezes maior para IMC $\geq 25 \mathrm{~kg} / \mathrm{m}^{2}$ ) e à história familiar de obesidade, mostrando a importância do excesso de gordura corporal na determinação da SM (6).

Portanto, apesar de as dúvidas a respeito da fisiopatologia e da melhor terapêutica para a LAHIV, a detecção dessa síndrome deve fazer parte do acompanhamento clínico dos pacientes infectados pelo HIV, tendo em vista sua alta prevalência (mais da metade dos indivíduos, na nossa casuística). Da mesma forma, a presença de SM deve ser ativamente pesquisada, visto que é tão ou mais comum em infectados por HIV quanto na população geral, visando à pronta instituição de medidas farmacológicas e não-farmacológicas para prevenção cardiovasculares nesses indivíduos de alto risco.

\section{REFERÊNCIAS}

1. Hadigan C, Meigs JB, Corcoran C, Rietschel P, Piecuch S, Basgoz N, et al. Metabolic abnormalities and cardiovascular disease risk factors in adults with human immunodeficiency virus infection and lipodystrophy. Clin Infect Dis. 2001;32:130-9.

2. Grinspoon S, Carr A. Cardiovascular risk and body-fat abnormalities in HIV-infected patients. N Engl J Med. 2005; 351:48-62.

3. Carr A. HIV lipodystrophy: risk factors, pathogenesis, diagnosis and management. AIDS. 2003;17 Suppl 1:S141-8.

4. Santos $C P$, Felipe $Y X$, Braga PE, Ramos D, Lima RO, Segurado AC. Self-perception of body changes in persons living with HIV/AIDS: prevalence and associated factors. AIDS. 2005; 19 Suppl 4:S14-21.

5. Grundy SM, Cleeman JI, Daniels SR, Donato KA, Eckel RH, Franklin BA, et al. Diagnosis and management of the metabolic syndrome: an American Heart Association/National Heart, Lung, and Blood Institute Scientific Statement. Circulation. 2005;112:2735-52. 
6. Estrada V, Martínez-Larrad MT, González-Sánchez JL, Villar NGP, Zabena C, Fernández $\mathrm{C}$, et al. Lipodystrophy and metabolic syndrome in HIV-infected patients treated with antiretroviral therapy. Metab Clin Exp 2006;55:940-5.

7. Jericó C, Knobel H, Montero M, Ordoñez-Llanos J, Guelar A, et al. Metabolic syndrome among HIV-infected patients. Diabetes Care. 2005;28:144-9.

8. Bonfanti P, Giannattasio C, Ricci E, Facchetti R, Rosella E, Franzetti $\mathrm{M}$, et al. HIV and metabolic syndrome: a comparison with the general population. J Acquir Immune Defic Syndr. 2007;45:426-31.

9. National Cholesterol Education Program (NCEP) Expert Panel on Detection, Evaluation, and Treatment of High Blood Cholesterol in Adults (Adult Treatment Panel III). Third Report of the National Cholesterol Education Program (NCEP) Expert Panel on Detection, Evaluation, and Treatment of High Blood Cholesterol in Adults (Adult Treatment Panel III) final report. Circulation. 2002;106:3143-421.

10. Alberti KGMM, Zimmet P, Shaw J. The metabolic syndrome: a new worldwide definition. Lancet. 2005;366:1059-62.

11. Brasil. Ministério da Saúde. Estimativa de prevalência do HIV na população brasileira de 15 a 49 anos, 2004 [acesso em 2007 Apr 24]. Disponível em: http://www.aids.gov.br/data/Pages/LUMIS72418C70PTBRIE.htm.

12. Chen D, Misra A, Garg A. Lipodystrophy in human immunodeficiency virus-infected patients. J Clin Endocrinol Metab. 2002;87:4845-56.

13. Instituto Brasileiro de Geografia e Estatística (IBGE). Estimativa da população - 2004 [acesso em 2007 Apr 24]. Disponível em: http://www.ibge.gov.br/home/estatistica/população/estimativa2004/estimativa.shtm.

14. Friis-Møller $N$, Weber R, Reiss $P$, Thiébault R, Kirk $O$, d'Arminio Monforte $A$, et al. Cardiovascular risk factors in HIV patients: association with antiretroviral therapy. AIDS. 2003;17:1179-93.

15. Friis-Møller N, Sabin CA, Weber R, d'Arminio Monforte A, ElSadr WM, Reiss $P$, et al. The Data Collection on Adverse Events of Anti-HIV Drugs (DAD) Study Group. Combination antiretroviral therapy and the risk of myocardial infarction. N Engl J Med. 2003;349:1993-2003.

16. Leow MKS, Addy CL, Mantzoros CS. Human immunodeficiency virus/highly active antiretroviral therapy-associated metabolic syndrome: clinical presentation, pathophysiology, and therapeutic strategies. J Clin Endocrinol Metab. 2003;88:1961-76.

17. Martinez E, Mocroft A, García-Viejo MA, Pérez-Cuevas JB Blanco JL, Mallolas J, et al. Risk of lipodystrophy in HIV-1-infected patients treated with protease inhibitors: a prospective cohort study. Lancet. 2001;357:592-8.

18. Pujari SN, Dravid A, Naik E, Bhagat S, Tash K, Nadler JP, et al. Lipodystrophy and dyslipidemia among patients taking firstline, World Health Organization-recommended highly active antiretroviral therapy regimens in Western India. J Acquir Immune Defic Syndr. 2005;39:199-202.

19. Carr A, Law M. An objective lipodystrophy severity grading scale derived from the lipodystrophy case definition score. $J$ Acquir Immune Defic Syndr. 2003;33:571-76.

20. Valente AMM, Reis AF, Machado DM, Succi RCM, Chacra AR. Alterações metabólicas da síndrome lipodistrófica do HIV. Arq Bras Endocrinol Metab 2005;49:871-81.

21. Lichtenstein KA, Ward DJ, Moorman AC, Delaney KM, Young B, Palella FJ Jr, et al. Clinical assessment of HIV-associated lipodystrophy in an ambulatory population. AIDS. 2001;15:1389-89.

22. Riddler SA, Smit E, Cole SR, Li R, Chmiel JS, Dobs A, et al. Impact of HIV infection and HAART on serum lipids in men. JAMA. 2003;289:2978-82.
23. Asztalos BF, Schaefer EJ, Horvath KV, Cox CE, Skinner S, Gerrior J, et al. Protease inhibitor-based HAART, HDL, and CHD risk in HIV-infected patients. Atherosclerosis. 2005;184:72-7.

24. Nolan D, Mallal S. Getting to the HAART of insulin resistance. AIDS. 2001;15:2037-41.

25. Brar I, Shuter J, Thomas A, Daniels E, Absalon J. A comparison of factors associated with prevalent diabetes mellitus among HIV-infected antiretroviral-naive individuals versus individuals in the National Health and Nutritional Examination Survey cohort. J Acquir Immune Defic Syndr. 2007;45:66-71.

26. Ministério da Saúde. 1988. Estudo multicêntrico sobre a prevalência do diabete melito no Brasil [acesso em 2007 June 23]. Disponível em: http://tabnet.datasus.gov.br/cgi/idb2006/d10. htm.

27. Guimarães MMM, Greco DB, Ribeiro-Oliveira A Jr, Penido MG Machado LJC. Distribuição da gordura corporal e perfis lipídico e glicêmico de pacientes infectados pelo HIV. Arq Bras Endocrinol Metab 2007:51:42-51.

28. Oliveira EP, Souza MLA, Lima MDA. Prevalência de síndrome metabólica em uma área rural do semi-árido baiano. Arq Bras Endocrinol Metab 2006;50:456-65.

29. Bruno R, Gazzaruso C, Sacchi P, Zocchetti C, Giordanetti S Garzaniti $A$, et al. High prevalence of metabolic syndrome among HIV-infected patients: link with the cardiovascular risk. J Acquir Immune Defic Syndr. 2002;31:363-5.

30. Samaras K, Wand H, Law M, Emery S, Cooper D, Carr A. Prevalence of metabolic syndrome in HIV-infected patients receiving highly active antiretroviral therapy using International Diabetes Foundation and Adult Treatment Panel III Criteria - associations with insulin resistance, disturbed body fat compartmentalization, elevated C-reactive protein, and hypoadiponectinemia. Diabetes Care. 2007;30:113-9.

31. Mondy K, Overton ET, Grubb J, Tong S, Seyfried W, Powderly W, et al. Metabolic syndrome in HIV-infected patients from an urban, midwestern US outpatient population. Clin Infect Dis. 2007;44:726-34.

32. Gami AS, Witt BJ, Howard DE, Erwin PJ, Gami LA, Somers VK, et al. Metabolic syndrome and risk of incident cardiovascular events and death - a systematic review and meta-analysis of longitudinal studies. J Am Coll Cardiol 2007;49:403-14.

33. van Wijk JPH, de Koning EJ, Cabezas MC, Joven J, op't Roodt J, Rabelink TJ, et al. Functional and structural markers of atherosclerosis in human immunodeficiency virus-infected patients. J Am Coll Cardiol. 2006;47:1117-23.

34. Bonfanti P, Ricci E, Socio G, Zeme D, Carradori S, Penco G, et al. Metabolic syndrome: a real threat for HIV-positive patients? Results from the SIMONE Study. J Acquir Immune Defic Syndr. 2006;42:128-30.

35. Noor MA, Seneviratne T, Aweeka FT, Lo JC, Schwarz JM, Mulligan $\mathrm{K}$, et al. Indinavir acutely inhibits insulin-stimulated glucose disposal in humans: a randomized, placebo-controlled study. AIDS. 2002;16:F1-8.

36. Murata $\mathrm{H}$, Hruz PW, Mueckler M. The mechanism of insulin resistance caused by HIV protease inhibitor therapy. J Biol Chem. 2000;275:20251-4.

\section{Endereço para correspondência:}

Departamento de Clínica Médica do Centro de Ciências da Saúde (CCS/UEL)

Leandro Arthur Diehl

Av. Robert Koch, 60

86038-350 Londrina, PR

E-mail: leandro@portalendocrino.com.br 